\title{
Periodontal manifestations in a patient with psoriasis: A case report
}

\author{
Kranti K, Mani R, Shaesta Begaum
}

\begin{abstract}
Introduction: It is well established that several inflammatory conditions exist together and at an increased incidence in patients with psoriasis. Psoriasis is a relatively common chronic immune mediated inflammatory disease, commonly involving the skin, which usually present as cutaneous erythematous papules and plaques covered by whitish scales. However, only few authors have reported oral involvement in this group of patients. Case Report: The present case report discusses the clinical presentation of periodontal findings and the importance of diagnosing the oral condition in any form of mucocutaneous disorders. Conclusion: An accurate diagnosis is dependent on a thorough clinical examination, biopsy of the oral lesions and a history of cutaneous psoriasis.
\end{abstract}

Keywords: Psoriasis, Oral lesions, Plaque like lesions, Biopsy and severe periodontitis

$* * * * * * * * *$

Kranti K, Mani R, Begaum S. Periodontal manifestations in a patient with psoriasis: A case report. International Journal of Case Reports and Images 2013;4(7):372-375.

\section{Kranti $\mathrm{K}^{1}$, Mani $\mathrm{R}^{1}$, Shaesta Begaum ${ }^{2}$}

Affiliations: ${ }^{1} \mathrm{MDS}$, Professor, Department of Periodontics, M.S Ramaiah Dental College, Bangalore; ${ }^{2}$ MDS, Former Post Graduate Student, Department of Periodontics, M.S Ramaiah Dental College, Bangalore.

Corresponding Author: Dr. Kranti K, MDS, Professor, Department of Periodontics, MSR Nagar, MSRIT Post, M.S Ramaiah Dental College, Bangalore - 560054; Ph: (0) 9880382393; Email: krantireddy@rediffmail.com

Received: 16 April 2012

Accepted: 02 July 2012

Published: 01 July 2013 doi:10.5348/ijcri-2013-07-334-8

\section{INTRODUCTION}

"What cannot be cured must be endured" -A 14th century proverb.

Psoriasis is a relatively common chronic inflammatory and proliferative dermatological disease characterized by epithelial hyperplasia presenting clinically as cutaneous erythematous papules and plaques covered by whitish scales commonly seen on the extensor dorsal cutaneous surfaces [1].

It has been described for 200 years and affects $2-3 \%$ of population worldwide. Its prevalence in India ranges from $0.5-5.6 \%$. Two peaks of age of onset are seen. Early onset psoriasis or type I psoriasis is seen between 15-25 years. The late onset psoriasis or type II psoriasis is seen between $57-60$ years of age [2]. A family history of psoriasis has been found in at least $35 \%$ of the affected patients [1]. In pediatric population females are more commonly affected but in adults slightly higher in males [3].

Although the etiology of psoriasis remains unclear, the epithelial changes are related to a defect in the control of keratinocyte proliferation characterized by increased proliferative activity of the cutaneous keratinocytes [4].

In contrast to cutaneous psoriasis, intraoral psoriasis has been rarely reported in literature. It is strange that the existence of oral psoriasis seems so rare. Other papulo squamous disorders such as lichen planus are frequently associated with oral manifestations, yet oral psoriasis is rare, given the prevalence of cutaneous disease.

In 1903, Oppenheim reported the first oral psoriatic lesions with confirmatory histological findings. In a study by Baker and Ryan (1968) involving more than 100 cases of cutaneous pustular psoriasis, only $5 \%$ showed corresponding oral lesion. In another study, 
Hietanen et al. (1984), reported a $2 \%$ incidence of intraoral lesions mostly occur in the presence of cutaneous disease [5]. Therefore, the diagnosis of psoriasis should usually include simultaneous presentation of oral and cutaneous disease. It usually occurs with white "plaque like" lesions, erythematous lesions or a mixed appearance, increased frequency of geographic tongue is seen but any gingival and periodontal changes appear to be the most obscure with only five reported evidence of periodontal disease [6].

Psoriasis may present with severe systemic involvement and in some cases may be fatal. However, it may also undergo spontaneous remissions for weeks, months or even years. Little information is currently available for the treatment of lesions associated with psoriasis.

This case report describes the clinical presentation of periodontal findings in a patient having cutaneous psoriasis.

\section{CASE REPORT}

A 45-year-old female presented to the Department of Periodontics, M.S. Ramaiah Dental College and Hospital, Bangalore, with a complaint of loosening of upper and lower front teeth since two months. It was associated with intermittent pain since two months. Her medical history revealed that she was a known case of psoriasis past 22 years. She was allergic to penicillin. Her family history was positive for psoriasis and she was on medication for same. Scaly type cutaneous lesions, whitish to red in color, were seen on the extensor aspect of forearms, legs, and also the scalp (Figures 1 and 2).
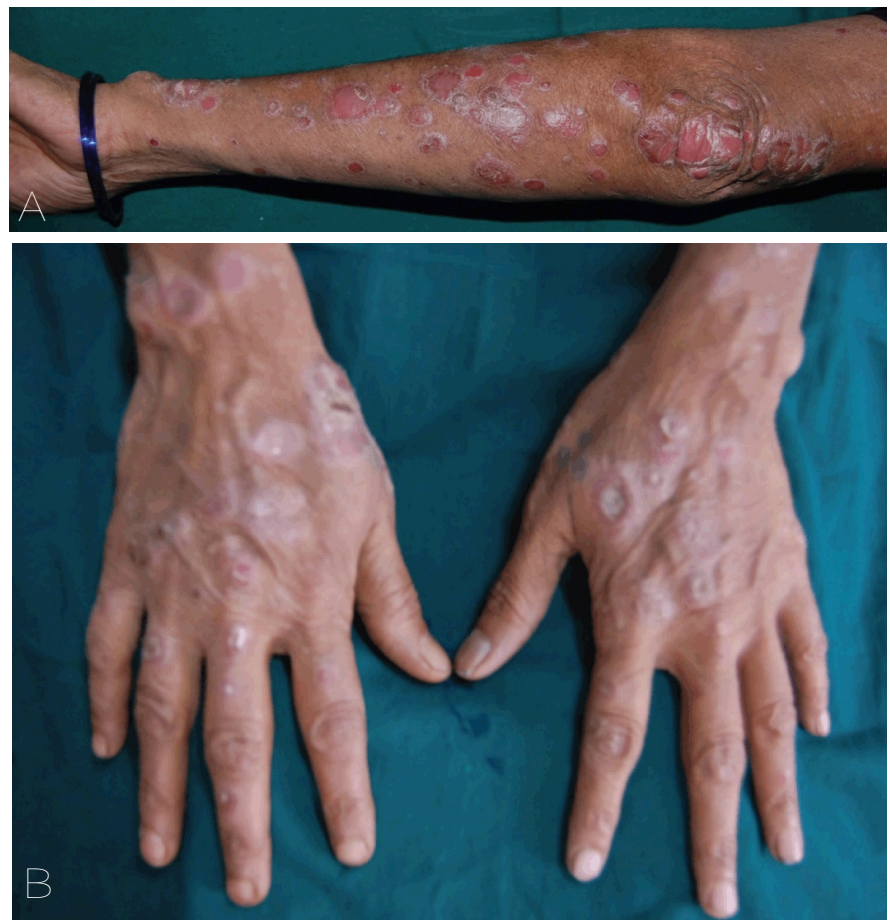

Figure 1: Scaly type cutaneous lesions (A) Dorsal aspect of forearm, (B) Dorsal aspect of hand.
An intraoral clinical examination presented missing teeth i.r.t 15, 31, 32, 44, 45 and 46. Oral hygiene was poor with increased local factors. There was generalized gingival inflammation with increased tendency to bleed on probing. Periodontal examination revealed probing depth of $8-10 \mathrm{~mm}$. All the remaining teeth were found to be in compromised periodontal health with generalized Grade III mobility. There was also generalized recession present (Figure 2).

Orthopantomogram revealed severe horizontal bone loss around all the teeth. Bone loss was greater than apical third of the roots of all the teeth. Grade III furcation i.r.t 16, 26 and grade IV furcation 47 (Figure 3). Blood investigations showed normal values except for erythrocyte sedimentation rate (ESR) which was high. A biopsy was obtained, Histopathologic features showed parakeratinized stratified epithelium with elongated tube like reteridges. Epithelium over the connective tissue papillae was thinned with the proximity of the vascularized stromal tissue. Intense pooling of chronic inflammatory cells was evident suggestive of psoriasis (Figure 4).

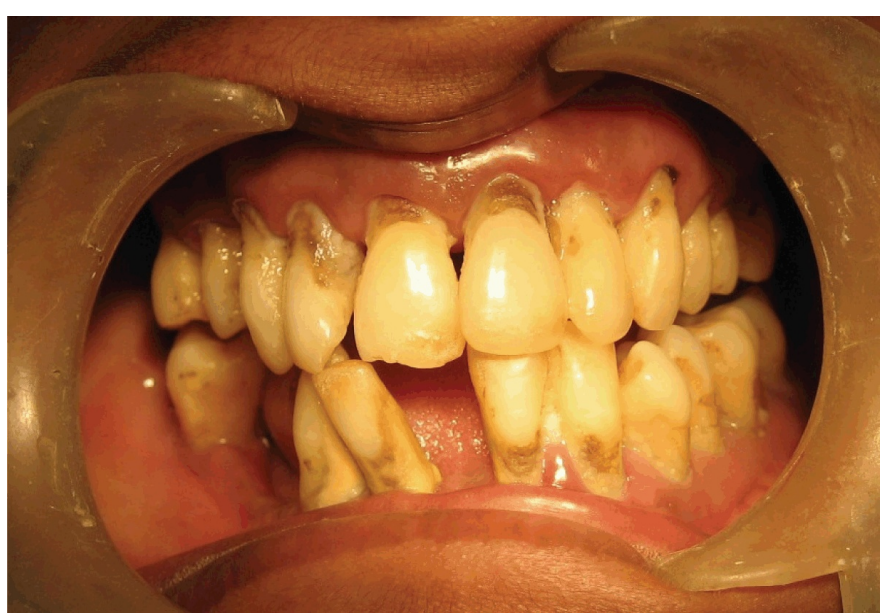

Figure 2: Frontal view of missing teeth with generalized recession.

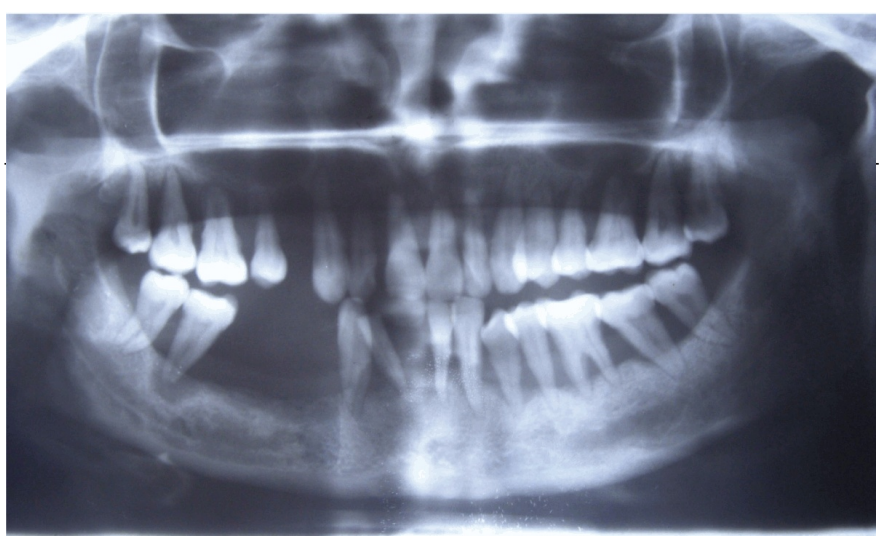

Figure 3: Orthopantomogram showing severe horizontal bone lors around all the teeth. 


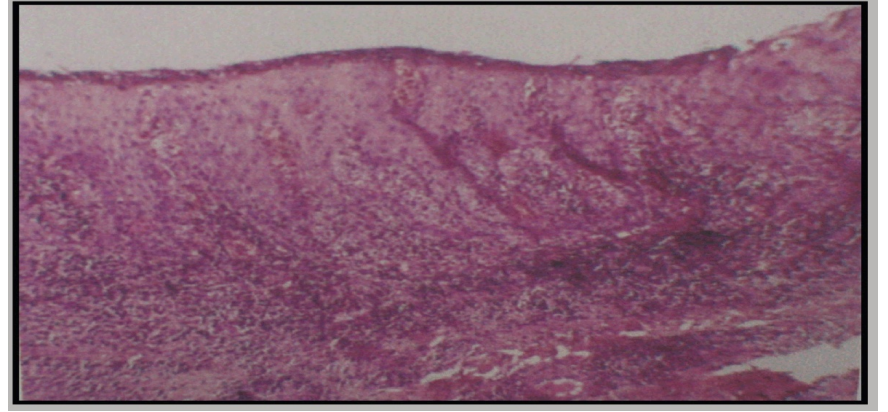

Figure 4: Biopsy showing parakeratinized stratified epithelium (H\&E stain, x100)

Based on the clinical and radiological appearance a diagnosis of aggressive periodontitis was made, in a known case of psoriasis. Patient was advised to undergo full mouth extraction followed by replacement. Patient was recalled and follow-up was done.

\section{DISCUSSION}

An intelligent person once stated "Beware the man with one case". Although it is true that no generalizations are to be made and no definite conclusions are to be drawn from a single event, some interesting observations can be made in this rather unique case.

A definitive diagnosis of psoriasis associated with periodontitis can pose a significant challenge for a clinician.

The marked gingival lesions observed in this case, along with severe destruction of the periodontal tissues affecting almost all the teeth. It is possible that this form of severe periodontitis could have been a coincidental finding, unrelated to psoriasis. However, literature suggest that several inflammatory conditions, exist comorbidly and at an increased incidence in patients with psoriasis [7]. This has been attributed to the common inflammatory pathways with similar pathogenic mechanism, involving cytokine dysregulation in psoriasis and several associated diseases such as arthritis, diabetes, cardiovascular disease and irritable bowel syndrome which are referred to as immune mediated inflammatory diseases $[7,8]$.

As illustrated by this case, a definitive diagnosis of oral psoriasis can pose a significant challenge for the clinican. Further, it is generally agreed that the major criterion in confirming a diagnosis of oral psoriasis is the presence of mucocutaneous lesions which has been so in the present case report.

The immune system has been strongly implicated in the pathogenesis of psoriasis that resembles a $\mathrm{T}$ cell mediated, autoimmune, inflammatory disease $[7,8]$. T cells are accompanied by increase number of dermal dendritic cells, macrophages and mast cells. It is thought that a stimulus such as trauma or infection which could be plaque induced periodontal infection triggers a plexus of cellular events by initiating a cascade of cytokines, creating an inflammatory response. Once activated dendritic antigen presenting cells and $\mathrm{T}$ cells release cytokines, chemokines and growth factors which trigger keratinocyte proliferation, altered differentiation and an angiogenic tumor response giving rise to psoriatic lesions.

There is presently no definitive treatment for psoriasis and managing the intraoral lesions is not well documented in literature. Spontaneous remission of these conditions have been reported. However, patient with persistant lesions usually require therapy directed at palliation of symptomatic lesions. This may be in form of topical anaesthetics, diphenhydramine hydrochloride and alkali rinses. However, treatment will only usually provide temporary relief of symptoms. This case report throws a light on the severity of periodontal disease associated with psoriatic lesions. Treatment goals are directed towards palliation of symptomatic lesions.

\section{CONCLUSION}

Although psoriasis is a common papulosquamous disease of the skin, oral psoriasis is very rare which could be attributed to cell turnover rate. Considering this rarity, this case can enlighten on the new insights on the pathogenesis of periodontal disease and its relevance on the severity of periodontal lesion which can alter the treatment goals, and further questions whether it is a cause or a consequence of disease severity need to be established.

$* * * * * * * * *$

\section{Author Contributions}

Kranti K - Substantial contributions to conception and design, Acquisition of data, Drafting the article, revising it critically for important intellectual content, Final approval of the version to be published

Mani R - Substantial contributions to conception and design, Analysis and interpretation of data, Drafting the article, Final approval of the version to be published

Shaesta Begaum - Substantial contributions to conception and design, Drafting the article, Revising it critically for important intellectual content, Final approval of the version to be published

\section{Guarantor}

The corresponding author is the guarantor of submission.

\section{Conflict of Interest}

Authors declare no conflict of interest.

\section{Copyright}

(C) Kranti $\mathrm{K}$ et al. 2012; This article is distributed under the terms of Creative Commons attribution 3.0 License which permits unrestricted use, distribution and reproduction in any means provided the original authors and original publisher are properly credited. (Please see 
www.ijcasereportsandimages.com/copyright-policy.php for more information.)

\section{REFERENCES}

1. Camp RDR. Chapter 35; Rook Textbook of dermatology. 2nd Edition.

2. Lebwohl Mark. Psoriasis. The Lancet 2003;361(9364):1197-204.

3. Younai FS, Phelan JA. Oral mucositis with feature of psoriasis; Report of a case and review of literature. Oral Surg Oral Med Oral pathol Oral Radiol Endod 1997 Jul;84(1):61-7.

4. Moschella L Samuel. Dermatology; 2nd Edition Vol 1, Page 504-8.
5. Bruce AJ, Rogers RS 3rd. Oral Psoriasis. Dermatol Clin 2003;21(1):99-104.

6. Brice MD, Danesh-Meyer MJ. Oral lesions in patients with Psoriasis: Clinical presentation and Management. J Periodontol 2000;71(12):1896-903.

7. Ulrich Mrowietz, James T Elder, Jonathan Barker. The importance of disease associations and concomitant therapy for the long-term management of psoriasis patients. Arch Dermatol Res 2006;298(7):309-19.

8. Brian J Nickoloff, Frank O Nestle. Recent insights into the immunopathogenesis of psoriasis provide new therapeutic opportunities. J Clin Invest 2004;113(12):1664-75.
Access full text article on other devices

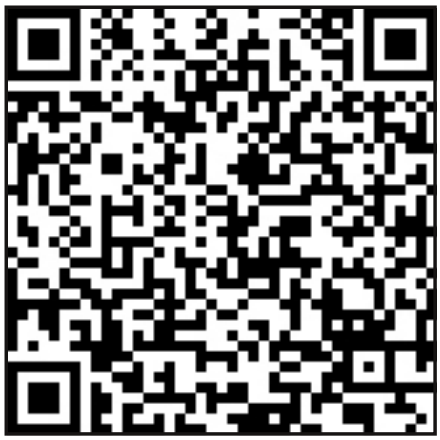

Access PDF of article on other devices

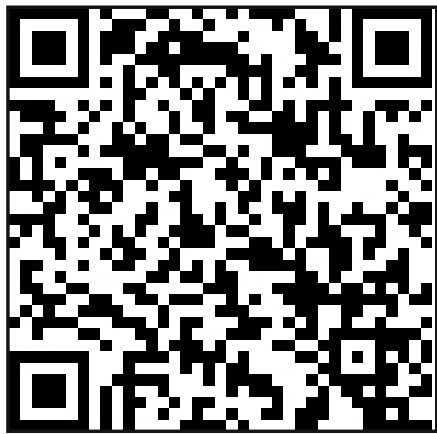

